TITLE:

\title{
A new point estimation method for statistical moments based on dimension-reduction method and direct numerical integration
}

$\operatorname{AUTHOR}(\mathrm{S})$ :

FAN, Wenliang; LIU, Runyu; ANG, Alfredo H. -S.; LI, Zhengliang

\section{CITATION:}

FAN, Wenliang ... [et al]. A new point estimation method for statistical moments based on dimension-reduction method and direct numerical integration. Japan-China Workshop on Analysis and Optimization of Large-scale Structures 2018: 9-9: A06.

ISSUE DATE:

2018-05-14

URL:

http://hdl.handle.net/2433/231239

RIGHT: 


\title{
A new point estimation method for statistical moments based on dimension-reduction method and direct numerical integration
}

\author{
Wenliang FAN ${ }^{*}, 2$, Runyu LIU ${ }^{1}$, Alfredo H-S ANG ${ }^{3}$, Zhengliang LI ${ }^{1,2}$ \\ ${ }^{1}$ School of Civil Engineering, Chongqing University, Chongqing 400045, China \\ (davidfwl@126.com) \\ ${ }^{2}$ Key Laboratory of New Technology for Construction of Cities in Mountain \\ Area(Chongqing University), Ministry of Education, Chongqing 400045, China \\ ${ }^{3}$ Department of Civil and Environmental Engineering, University of California, Irvine, \\ CA92697, USA
}

Keywords: statistical moments, point estimate method, dimension-reduction method, high dimensional integration, curse of dimension

\begin{abstract}
Estimation of statistical moments of structural response is one of the main topics for analysis of random systems. The balance between accuracy and efficiency remains a challenge. After investigating of the existing point estimation methods (PEM) [1,2], a new point estimate method based on the dimension-reduction method (DRM) is presented. Firstly, by introducing transformations, a system with general variables is transformed into the one with independent variables. Secondly, the existing PEMs based on the DRMs are investigated. Thirdly, based on the qualitative analysis of difference in the approximations for response function and moment function, a new PEM is proposed, in which the response function is decomposing directly and the moments are calculated by high dimensional integral directly. By comparing with the existing PEM based on univariate DRM, the proposed method is more friendly and easier to implement without loss of accuracy and efficiency; as compared with the PEM based on the generalized DRM, the proposed method is of better precision at the cost of nearly the same efficiency and computational complexity, further, it does hold that the even-order moments are nonnegative. Finally, several examples are investigated to verify the performance of the new method.
\end{abstract}

\section{References}

[1] S. Rahman, H. Xu, A univariate dimension-reduction method for multidimensional integration in stochastic mechanics, Probab. Eng. Mech. 19 (4) (2004) 393-408.

[2] $\mathrm{H} . \mathrm{Xu}, \mathrm{S}$. Rahman, A generalized dimension-reduction method for multidimensional integration in stochastic mechanics, Int. J. Numer. Methods Eng. 61 (12) (2004) 1992-2019. 